\title{
A U-shaped association between the LDL- cholesterol to HDL-cholesterol ratio and all- cause mortality in elderly hypertensive patients: a prospective cohort study
}

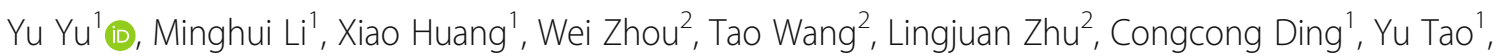
Huihui Bao ${ }^{1,2^{*}}$ and Xiaoshu Cheng ${ }^{1,2}$

\begin{abstract}
Background: The low-density lipoprotein cholesterol/high-density lipoprotein- cholesterol (LDL-C/HDL-C) ratio is an excellent predictor of cardiovascular disease (CVD). However, previous studies linking the LDL-C/HDL-C ratio to mortality have yielded inconsistent results and been limited by short follow-up periods. Therefore, the aim of the present study was to determine whether the LDL-C/HDL-C ratio could be an effective predictor of all-cause mortality in elderly hypertensive patients.

Methods: A total of 6941 hypertensive patients aged 65 years or older who were not treated with lipid-lowering drugs were selected from the Chinese Hypertension Registry for analysis. The endpoint of the study was all-cause mortality. The relationship between the LDL-C/HDL-C ratio and all-cause mortality was determined using multivariate Cox proportional hazards regression, smoothing curve fitting (penalized spline method), subgroup analysis and Kaplan-Meier survival curve analysis.
\end{abstract}

Results: During a median follow-up of 1.72 years, 157 all-cause deaths occurred. A U-shaped association was found between the LDL-C/HDL-C ratio and all-cause mortality. Patients were divided according to the quintiles of the LDL-C/HDL-C ratio. Compared to the reference group (Q3: 1.67-2.10), patients with both lower (Q1 and Q2) and higher (Q4 and Q5) LDL-C/HDL-C ratios had higher all-cause mortality (<1.67: HR 1.81, 95\% Cl: 1.08-3.03; $\geq 2.10$ : HR 2.00, 95\% Cl: 1.18-3.39). Compared with the lower and higher LDL-C/HDL-C ratio groups, patients with LDL-C/HDL$C$ ratios of $1.67-2.10$ had a significantly higher survival probability (log-rank $P=0.038$ ).

Conclusions: The results suggest that there is a U-shaped association between the LDL-C/HDL-C ratio and all-cause mortality. Both lower and higher LDL-C/HDL-C ratios were associated with increased all-cause mortality in elderly hypertensive patients.

Keywords: Low-density lipoprotein cholesterol to high-density lipoprotein cholesterol ratio, U-shaped curve, Mortality, Hypertension, Low-density lipoprotein cholesterol, High-density lipoprotein cholesterol, Cohort study

\footnotetext{
* Correspondence: huihui_bao77@126.com

'Department of Cardiovascular Medicine, the Second Affiliated Hospital of Nanchang University, No. 1 Minde Road, Nanchang 330006, Jiangxi, China ${ }^{2}$ Center for Prevention and Treatment of Cardiovascular Diseases, the Second Affiliated Hospital of Nanchang University, Nanchang, Jiangxi, China
}

(c) The Author(s). 2020 Open Access This article is licensed under a Creative Commons Attribution 4.0 International License, which permits use, sharing, adaptation, distribution and reproduction in any medium or format, as long as you give appropriate credit to the original author(s) and the source, provide a link to the Creative Commons licence, and indicate if changes were made. The images or other third party material in this article are included in the article's Creative Commons licence, unless indicated otherwise in a credit line to the material. If material is not included in the article's Creative Commons licence and your intended use is not permitted by statutory regulation or exceeds the permitted use, you will need to obtain permission directly from the copyright holder. To view a copy of this licence, visit http://creativecommons.org/licenses/by/4.0/ The Creative Commons Public Domain Dedication waiver (http://creativecommons.org/publicdomain/zero/1.0/) applies to the data made available in this article, unless otherwise stated in a credit line to the data. 


\section{Background}

The leading causes of death worldwide include stroke and cardiovascular disease (CVD), both of which are associated with higher levels of low-density lipoprotein cholesterol (LDL-C) and lower levels of high-density lipoprotein cholesterol (HDL-C) [1, 2]. The LDL-C/HDL-C ratio, which is a new marker, is calculated by dividing LDL-C by HDL-C. Enomoto $\mathrm{M}$ et al. [3] followed 1920 Japanese individuals for 8 years, and found that the LDL-C/HDL-C ratio had greater predictive value for carotid intima-media thickness than LDL-C or HDL-C alone. Ridker PM et al. [4] followed 15,632 US women for 10 years and found that compared with LDL-C or HDL-C, the LDL-C/HDL-C ratio had a stronger relationship with the CVD composite endpoint, which included myocardial infarction, stroke, coronary revascularization, and cardiovascular-related death. CVD and death are closely related, and the LDL-C/HDL-C ratio should also be suitable for predicting death. However, previous studies on the LDL-C/HDL-C ratio have drawn inconsistent conclusions. Some studies have suggested that the LDL-C/HDL-C ratio is positively associated with CVD [5-8], while others have found a negative correlation between the LDL-C/HDL-C ratio and all-cause mortality $[9,10]$. The reasons for these inconsistencies can be attributed to differences in the study population, follow-up, and study endpoints. Furthermore, the above studies proposed different ranges of the $\mathrm{LDL}-\mathrm{C} / \mathrm{HDL}-\mathrm{C}$ ratio that were associated with the lowest risk of CVD or all-cause mortality. These factors prevent the LDL-C/HDL-C ratio from being a valuable predictor of all-cause mortality in clinical practice.

In addition, there have been some limitations of the studies on the relationship between LDL-C/HDL-C ratio and all-cause mortality, including small sample sizes, short follow-up durations, and the varying disease severity; therefore, the relationship between the LDL-C/HDL-C ratio and all-cause mortality remains unclear. Notably, elderly hypertensive patients (defined as hypertensive patients over the age of 65 years) have higher mortality than the rest of the population [11]. Therefore, it is necessary for researchers to identify a clinically valuable predictor of all-cause mortality in elderly patients with hypertension.

In an effort to address the significant gaps in knowledge, the present study was performed to investigate the potential relationship of the LDL-C/HDL-C ratio with all-cause mortality, and to examine the optimal range of the LDL-C/HDL-C ratio in the hypertensive population aged 65 years or older, using data from the China Hypertension Registry Study.

\section{Methods}

\section{Study design and participants}

The study data were drawn from the China Hypertension Registry Study (http://www.chictr.org.cn/, No:
ChiCTR1800017274). Details of the methodology, and primary objectives of this study had been described elsewhere [12]. The inclusion and exclusion criteria for the study are described in detail in Table S1. In short, this study is an observational, real-world investigation of hypertension. Hypertension was defined by a systolic blood pressure (SBP) $\geq 140 \mathrm{mmHg}$ and/or diastolic BP (DBP) $\geq 90 \mathrm{mmHg}$, self-reported history of hypertension, or the use of antihypertensive $\operatorname{drug}(\mathrm{s})$ at baseline [13]. From March 2018 to August 2018, 14,268 patients with hypertension were recruited in Wuyuan, Jiangxi Province, China, and the treatment, related risk factors and prognosis of patients with hypertension were evaluated. In our study, we selected 6941 hypertensive patients aged 65 years or older who were not treated with lipid-lowering drugs from the China Hypertension Registry Study. The study protocol was approved by the Ethics Committee of the Anhui Medical University Biomedical Institute (No. CH1059), and all participants signed informed consent.

\section{Data collection and outcome definition}

All study participants had fasting, venous blood samples collected by trained study staff during the baseline data collection period. Total cholesterol (TC, mmol/L), triglycerides (TG, mmol/L), LDL-C (mmol/L), HDL-C $(\mathrm{mmol} / \mathrm{L})$, serum uric acid (SUA, $\mu \mathrm{mol} / \mathrm{L})$, the estimated glomerular filtration rate (eGFR, $\left.\mathrm{ml} / \mathrm{min} / 1.73 \mathrm{~m}^{2}\right)$, homocysteine (Hcy, $\mu \mathrm{mol} / \mathrm{L})$, fasting blood glucose (FBG, $\mathrm{mmol} / \mathrm{L})$ and albumin $(\mathrm{g} / \mathrm{L})$ were measured by an automatic clinical analyzer (Beckman Coulter, USA) in Biaojia Biotechnology Laboratory, Shenzhen, China [14]. The LDL-C/HDL-C ratio was calculated by dividing LDL-C by HDL-C. In addition to the abovementioned laboratory indicators, other covariates in the study included age (years), sex (male or female), body mass index (BMI, $\mathrm{kg} / \mathrm{m}^{2}$ ), smoking status (never, former and current), alcohol consumption, history of disease (including stroke, CVD and diabetes), systolic/diastolic blood pressure (mmHg) and drug history (including antihypertensive drugs, antiplatelet drugs and glucose-lowering drugs).

The outcome of our study was all-cause mortality from 31 August 2018 to 31 March 2020. Causes of death included stroke, CVD, tumor respiratory diseases, and other reasons. All-cause mortality was ascertained from the Local Healthcare Security Administration, Centers for Disease Control and Prevention, and hospitals.

\section{Statistical analysis}

Study population characteristics at baseline are presented stratified by the quintiles of the LDL-C/HDL-C ratio. Data are presented as the means \pm SDs or proportions. The Mann-Whitney test was used to identify significant differences between the two groups. Multiple Cox proportional hazards regression analysis was used 
to analyze the relationship between the LDL-C/HDL-C ratio and all-cause mortality, and the results are presented as hazard ratios (HRs) and $95 \%$ confidence intervals (CIs). The adjusted variables were selected based on their clinical importance, statistical significance in the univariable analysis, and potential confounding, indicated by estimates that individually changed by at least $10 \%$. Fully adjusted smoothing curve fitting (penalized spline method) visually showed the relationship between the LDL-C/HDL-C ratio and all-cause mortality. Stratified analyses were conducted, with stratification by sex, BMI, stroke, CVD, diabetes and eGFR, to identify potential subgroups in which there was a significant association between the LDL-C/HDL-C ratio and all-cause mortality. Survival was estimated by the Kaplan-Meier method, and any differences in survival were evaluated with stratified log-rank tests.

All data analysis and form generation were performed using the statistical package R (http://www.R-project.org, The $\mathrm{R}$ Foundation) and Empower (R) (www.empower stats.com; X\&Y Solutions, Inc., Boston, MA). When a two-tailed $P$ was $<0.05$, we had sufficient reason to believe that the results were statistically significant.

\section{Results}

\section{Baseline characteristics of the study participants}

A total of 6941 hypertensive patients aged 65 years or older who were not treated with lipid-lowering drugs were selected for the final data analysis (mean age: $71.20 \pm 5.30$; male: $48.18 \%$ ). The distribution of the baseline population characteristics according to the baseline LDL-C/HDL-C ratio quintiles is described in Table 1. Compared with the lower groups (Q1 and Q2), the higher groups (Q4 and Q5) had higher values of DBP, TC, TG, LDL-C, FBG, albumin, and SUA; were more likely to have stroke and diabetes; and were more likely to take antihypertensive drugs and glucose-lowering drugs. In contrast, the higher groups $(\mathrm{Q} 4$ and Q5) were younger; less likely to be male; less likely to smoke or consume alcohol; and had lower values of HDL-C and the eGFR (all $P<0.05$ ).

\section{U-shaped relationship between LDL-C/HDL-C ratio and all-cause mortality}

In the present study, the average follow-up duration was 1.72 years, and a total of 157 all-cause deaths $(2.26 \%)$ occurred during the follow-up period. Table 2 shows the HR and $95 \% \mathrm{CI}$ values of the relationship between the LDL-C/HDL-C ratio and all-cause mortality in the crude model, model 1 (partially adjusted) and model 2 (fully adjusted). Multiple Cox proportional hazard regression analysis was used to examine the relationship between the LDL-C/HDL-C ratio and all-cause mortality. When the LDL-C/HDL-C ratio was used as a continuous variable, there was no significant relationship between the LDL-C/HDL-C ratio and all-cause mortality in any of the three models (all $P>0.05$ ). Then, the LDL-C/HDL-C ratio was converted from a continuous variable to a categorical variable. The patients were divided into five groups according to the quintiles of the LDL-C/HDL-C ratio. Compared to the reference group (Q3: 1.67-2.10), patients in both the lower (Q1 and Q2: $<1.67)$ and higher (Q4 and Q5: $\geq 2.10$ ) LDL-C/HDL-C ratio groups had higher mortality in all models (all $P<0.05$ ). In model 2 (fully adjusted model), both the low LDL-C/HDL-C ratio and high LDL-C/HDL-C ratio groups had relatively higher mortality (<1.67: HR 1.81, 95\% CI: $1.08-3.03$; $\geq 2.10$ : HR 2.00, 95\% CI: 1.18-3.39). In addition, the regression coefficients $(\beta)$ were added to the regression model (Table S3). These results suggest that the relationship between the LDL-C/HDL-C ratio and all-cause mortality is likely to be nonlinear. The fully adjusted smooth curve fitting showed a U-shaped relationship between the baseline LDL-C/ HDL-C ratio and all-cause mortality (Fig. 1).

As shown in Table 3, stratified analyses were conducted with stratification by sex, BMI, stroke, CVD, diabetes, eGFR, smoking and alcohol consumption. The U-shaped association was consistently observed in all subgroups, and there was no significant interaction between the LDL$\mathrm{C} / \mathrm{HDL}-\mathrm{C}$ ratio and all-cause mortality in any subgroup (all $P$ for interaction $>0.05$ ). The regression coefficients $(\beta)$ were added to the subgroup analysis model (Table S4).

The Kaplan-Meier survival curve for all-cause mortality stratified by LDL-C/HDL-C ratio levels is shown in Fig. 2. Compared with the lower and higher LDL-C/ HDL-C ratio groups, patients with LDL-C/HDL-C ratios of $1.67-2.10$ had a significantly higher survival probability $(\log$-rank $P<0.05)$.

\section{Discussion}

In the current study, we found a U-shaped relationship between the LDL-C/HDL-C ratio and all-cause mortality in the elderly hypertensive population in China. Both lower and higher LDL-C/HDL-C ratios were associated with higher all-cause mortality. The optimal range of the LDL-C/HDL-C ratio was 1.67-2.10. Compared with the lower and higher LDL-C/HDL-C ratio groups, patients with LDL-C/HDL-C ratios of $1.67-2.10$ had a significantly higher survival probability.

Although CVD is one of the leading causes of death in elderly patients with hypertension, studies on the relationship between the LDL-C/HDL-C ratio and all-cause mortality have been limited. Therefore, we also analyzed the LDL-C/HDL-C ratio and CVD, to thoroughly investigate the predictive value of the LDL-C/HDL-C ratio. Gragnano, $\mathrm{F}$ et al. [15] described the importance of lowering LDL-C to reduce the risk of CVD. However, studies have yielded inconsistent conclusions regarding the 
Table 1 Baseline Characteristics of the Cohort Per Quintiles of the LDL-C/HDL-C ratio

\begin{tabular}{|c|c|c|c|c|c|c|}
\hline \multirow[t]{2}{*}{ Characteristics* } & \multicolumn{5}{|c|}{ Quintiles of the LDL-C/HDL-C ratio } & \multirow[t]{2}{*}{$P$ value } \\
\hline & $\mathrm{Q} 1(<1.16)$ & Q2 (1.16-1.67) & Q3 (1.67-2.10) & Q4 (2.10-2.79) & Q5 ( $\geq 2.79)$ & \\
\hline $\mathrm{N}$ & 1387 & 1389 & 1389 & 1387 & 1389 & \\
\hline \multicolumn{7}{|l|}{ Demographics } \\
\hline Age, years & $71.75 \pm 5.51$ & $71.67 \pm 5.59$ & $71.10 \pm 5.36$ & $70.78 \pm 5.06^{c f}$ & $70.69 \pm 4.89^{\mathrm{dg}}$ & $<0.001$ \\
\hline Male, \% & $836(60.27)$ & $691(49.75)^{a}$ & $629(45.28)^{b}$ & $581(41.89){ }^{c f}$ & $607(43.70)^{d}$ & $<0.001$ \\
\hline $\mathrm{BMl}, \mathrm{kg} / \mathrm{m}^{2}$ & $20.85 \pm 4.95$ & $21.91 \pm 3.26^{\mathrm{a}}$ & $22.77 \pm 3.07^{\text {be }}$ & $23.56 \pm 3.25^{\mathrm{chh}}$ & $24.15 \pm 3.18^{\text {dgij }}$ & $<0.001$ \\
\hline Smoking, \% & & & & & & $<0.001$ \\
\hline Never & $638(46.00)$ & $732(52.74)$ & $778(56.01)$ & $788(56.85)$ & $768(55.33)$ & \\
\hline Former & $263(18.96)$ & $258(18.59)$ & $283(20.37)$ & $260(18.76)$ & $259(18.66)$ & \\
\hline Current & $486(35.04)$ & $398(28.67)^{a}$ & $328(23.61)^{b}$ & $338(24.39)^{c}$ & $361(26.01)^{d}$ & \\
\hline Alcohol consumption, $\%$ & $473(34.10)$ & $297(21.40)^{a}$ & $241(17.35)^{b}$ & $222(16.02) \mathrm{cf}$ & $199(14.34) d g$ & $<0.001$ \\
\hline \multicolumn{7}{|l|}{ History of disease, $\%$} \\
\hline Stroke & $74(5.34)$ & $78(5.62)$ & $90(6.48)$ & $90(6.49)$ & $130(9.36)^{d}$ & 0.001 \\
\hline CVD & $75(5.41)$ & $95(6.84)$ & $86(6.19)$ & $89(6.42)$ & $89(6.41)$ & 0.620 \\
\hline Diabetes & $158(11.39)$ & $180(12.96)$ & $206(14.83)$ & $274(19.75) \mathrm{cf}$ & $354(25.49)$ dgi & $<0.001$ \\
\hline \multicolumn{7}{|l|}{ Blood pressure } \\
\hline Systolic BP, mmHg & $149.30 \pm 18.83$ & $149.53 \pm 18.69$ & $150.66 \pm 17.87$ & $150.47 \pm 18.18$ & $150.33 \pm 18.47$ & 0.199 \\
\hline Diastolic BP, mmHg & $84.88 \pm 10.86$ & $85.22 \pm 10.71$ & $85.77 \pm 10.22$ & $85.32 \pm 10.07$ & $86.04 \pm 10.07$ & 0.030 \\
\hline \multicolumn{7}{|l|}{ Lipids } \\
\hline $\mathrm{TC}, \mathrm{mmol} / \mathrm{L}$ & $4.68 \pm 0.95$ & $4.95 \pm 0.97^{\mathrm{a}}$ & $5.15 \pm 1.03$ be & $5.37 \pm 1.07^{\text {ch }}$ & $5.68 \pm 1.19$ dgij & $<0.001$ \\
\hline $\mathrm{TG}, \mathrm{mmol} / \mathrm{L}$ & $0.98 \pm 0.44$ & $1.24 \pm 0.61^{\mathrm{a}}$ & $1.47 \pm 0.72$ be & $1.89 \pm 1.03$ ch & $2.37 \pm 1.34 \mathrm{dgij}$ & $<0.001$ \\
\hline LDL-C, mmol/L & $2.21 \pm 0.53$ & $2.68 \pm 0.55^{a}$ & $2.98 \pm 0.62$ be & $3.24 \pm 0.67^{\text {ch }}$ & $3.64 \pm 0.78^{\mathrm{dgij}}$ & $<0.001$ \\
\hline $\mathrm{HDL}-\mathrm{C}, \mathrm{mmol} / \mathrm{L}$ & $2.03 \pm 0.46$ & $1.74 \pm 0.36^{\mathrm{a}}$ & $1.59 \pm 0.33$ be & $1.45 \pm 0.30^{\mathrm{cfh}}$ & $1.27 \pm 0.27$ dgij & $<0.001$ \\
\hline LDL-C/HDL-C ratio & $1.10 \pm 0.20$ & $1.54 \pm 0.10^{a}$ & $1.88 \pm 0.10$ be & $2.24 \pm 0.11 \mathrm{cfh}$ & $2.89 \pm 0.41 \mathrm{dgij}$ & $<0.001$ \\
\hline \multicolumn{7}{|l|}{ Other plasma parameters } \\
\hline $\mathrm{Hcy}, \mu \mathrm{mol} / \mathrm{L}$ & $19.10 \pm 11.19$ & $19.32 \pm 11.28$ & $18.91 \pm 11.10$ & $19.18 \pm 11.94$ & $19.72 \pm 12.54$ & 0.439 \\
\hline $\mathrm{FBG}, \mathrm{mmol} / \mathrm{L}$ & $5.90 \pm 1.23$ & $6.00 \pm 1.35$ & $6.06 \pm 1.64$ & $6.19 \pm 1.46^{\text {cf }}$ & $6.43 \pm 1.81 \mathrm{dgij}$ & $<0.001$ \\
\hline Albumin, $g / L$ & $45.57 \pm 4.27$ & $45.83 \pm 4.06$ & $45.90 \pm 3.98$ & $46.18 \pm 3.89^{c}$ & $45.98 \pm 3.96$ & 0.002 \\
\hline$S \cup A, \mu \mathrm{mol} / \mathrm{L}$ & $413.30 \pm 123.79$ & $411.09 \pm 114.47$ & $415.57 \pm 118.22$ & $429.92 \pm 122.14{ }^{\text {cf }}$ & $450.71 \pm 120.26^{\text {dgij }}$ & $<0.001$ \\
\hline $\mathrm{eGFR}, \mathrm{ml} / \mathrm{min} / 1.73 \mathrm{~m}^{2}$ & $81.52 \pm 19.56$ & $81.51 \pm 18.15$ & $81.46 \pm 19.33$ & $80.44 \pm 19.26$ & $78.26 \pm 20.21 \mathrm{dgi}$ & $<0.001$ \\
\hline \multicolumn{7}{|l|}{ Medication use, \% } \\
\hline Antihypertensive drugs & $874(63.01)$ & $897(64.63)$ & $959(69.04)$ & $948(68.40)$ & $983(70.82)^{d}$ & $<0.001$ \\
\hline Antiplatelet drugs & $34(2.45)$ & $33(2.38)$ & $38(2.74)$ & $43(3.10)$ & $39(2.81)$ & 0.773 \\
\hline Glucose-lowering drugs & $34(2.45)$ & $47(3.38)$ & $56(4.03)$ & $85(6.13)^{c}$ & $100(7.20)^{d g}$ & $<0.001$ \\
\hline
\end{tabular}

Abbreviations: BMI body mass index; CVD cardiovascular disease; TC total cholesterol; TG triglyceride; $L D L-C$ low density lipoprotein cholesterol; $H D L-C$ high density lipoprotein cholesterol; SUA serum uric acid; eGFR estimated glomerular filtration rate; Hcy homocysteine; FBG fasting blood glucose

*Data are presented as number (\%) or mean \pm standard deviation

${ }^{a}$ indicates a significant difference between $\mathrm{Q} 2$ and $\mathrm{Q} 1, P<0.001 ;{ }^{\mathrm{b}}$ indicates a significant difference between $\mathrm{Q} 3$ and $\mathrm{Q} 1, P<0.001 ;{ }^{\mathrm{c}}$ indicates a significant difference between Q4 and Q1, $P<0.001 ;{ }^{\mathrm{d}}$ indicates a significant difference between Q5 and Q1, $P<0.001 ;{ }^{\mathrm{e}}$ indicates a significant difference between Q3 and $\mathrm{Q} 2, P<0.001 ;{ }^{\mathrm{f}}$ indicates a significant difference between Q4 and Q2, $P<0.001 ;{ }^{9}$ indicates a significant difference between $\mathrm{Q} 5$ and $\mathrm{Q} 2, P<0.001 ;{ }^{\mathrm{h}}$ indicates a significant difference between $Q 4$ and $Q 3, P<0.001 ;{ }^{i}$ indicates a significant difference between $Q 5$ and $Q 3, P<0.001 ;{ }^{j}$ indicates a significant difference between $\mathrm{Q} 5$ and $\mathrm{Q} 4, \mathrm{P}<0.001$

predictive value of the LDL-C/HDL-C ratio. Matsumoto I et al. [6] analyzed 687 patients who underwent PCI (mean age $67.7 \pm 9.9$ years, mean follow-up years $=2.75$ years) and found a positive association between the LDL-C/HDL-C ratio and CVD. They suggested that the
LDL-C/HDL-C ratio should be controlled below 1.5. Zhong et al. [7] enrolled 1937 acute coronary syndrome (ACS) patients (mean age 64.0 \pm 10.8 years, mean followup $=1.00$ years), and found that a high LDL-C/HDL-C ratio was associated with an increased risk of CVD. They 
Table 2 Association between the LDL-C/HDL-C ratio and all-cause mortality during the follow-up period

\begin{tabular}{|c|c|c|c|c|c|c|c|}
\hline \multirow[t]{2}{*}{ LDL-C/HDL-C ratio } & \multirow[t]{2}{*}{ Events, \% } & \multicolumn{2}{|l|}{ Crude model } & \multicolumn{2}{|l|}{ Model 1} & \multicolumn{2}{|l|}{ Model 2} \\
\hline & & $\mathrm{HR}(95 \% \mathrm{Cl})^{a}$ & $P$ value & HR $(95 \% \mathrm{Cl})$ & $\overline{P \text { value }}$ & HR (95\% Cl) & $P$ value \\
\hline Continuous & 157/6947 (2.29) & $0.93(0.73,1.19)$ & 0.558 & $1.05(0.83,1.34)$ & 0.674 & $1.02(0.76,1.36)$ & 0.912 \\
\hline \multicolumn{8}{|l|}{ Quintiles } \\
\hline$<1.16$ & 40/1387 (2.88) & $2.12(1.23,3.67)$ & 0.007 & $1.88(1.09,3.25)$ & 0.024 & $1.98(1.13,3.46)$ & 0.017 \\
\hline $1.16-1.67$ & $32 / 1389(2.30)$ & $1.69(0.96,2.98)$ & 0.070 & $1.57(0.89,2.78)$ & 0.118 & $1.64(0.92,2.90)$ & 0.091 \\
\hline $1.67-2.10$ & 19/1389 (1.37) & Reference & & Reference & & Reference & \\
\hline $2.10-2.79$ & 30/1387 (2.16) & $1.59(0.89,2.82)$ & 0.115 & $1.75(0.98,3.11)$ & 0.057 & $1.80(1.01,3.22)$ & 0.048 \\
\hline$\geq 2.79$ & 36/1389 (2.59) & $1.91(1.09,3.33)$ & 0.023 & $2.12(1.21,3.70)$ & 0.008 & $2.24(1.24,4.03)$ & 0.008 \\
\hline \multicolumn{8}{|l|}{ Categories } \\
\hline$<1.67$ & 72/2776 (2.59) & $1.91(1.15,3.16)$ & 0.012 & $1.73(1.04,2.87)$ & 0.034 & $1.81(1.08,3.03)$ & 0.024 \\
\hline $1.67-2.10$ & 19/1389 (1.37) & Reference & & Reference & & Reference & \\
\hline$\geq 2.10$ & $66 / 2776(2.38)$ & $1.75(1.05,2.91)$ & 0.032 & $1.93(1.16,3.23)$ & 0.011 & $2.00(1.18,3.39)$ & 0.010 \\
\hline$P$ for trend & & 0.570 & & 0.598 & & 0.767 & \\
\hline
\end{tabular}

${ }^{\text {a }}$ Cox proportional hazards models were used to estimate hazard ratio (HR) and 95\% confidence interval $(95 \% \mathrm{Cl})$

Abbreviations: $H R$ hazard ratio; $C l$ confidence interval; $L D L-C$ low density lipoprotein-cholesterol; $H D L-C$ high-density lipoprotein cholesterol

Model 1: adjusted for none. Model 2: adjusted for age, sex. Model 3: adjuste d for age, sex, BMI, SBP, DBP, TG, Hcy, FBG, SUA, eGFR, smoking, alcohol

consumption, diabetes, stroke, CVD and anti-hypertensive drugs

suggested that the LDL-C/HDL-C ratio should be controlled below 2.7. Yokokawa et al. [5] included 8714 male patients (mean age $63.7 \pm 11.5$ years, mean followup $=2.7 \pm 0.9$ years) and found that compared with patients with an LDL-C/HDL-C ratio $<2.6$, patients with a ratio $>2.6$ had a higher risk of CVD. The findings of the above studies suggest that there may be a positive association between the LDL-C/HDL-C ratio and CVD, and different studies have proposed different reference ranges for the LDL-C/HDL-C ratio. In contrast, You et al. [9] included 356 patients with intracranial hemorrhage (mean age $64.1 \pm 13.7$ years, mean follow-up $=0.22$ years) and found that the LDL-C/HDL-C ratio was negatively correlated with all-cause mortality, they

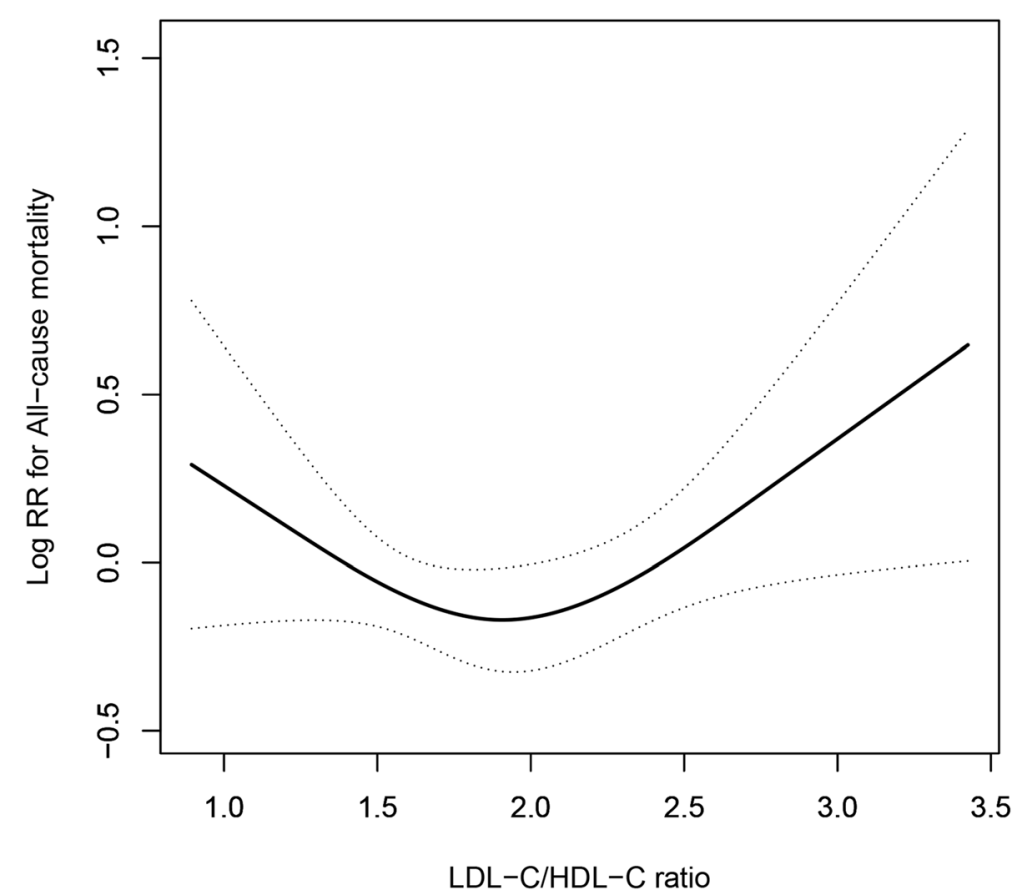

Fig. 1 Dose-response relationship between the LDL-C/HDL-C and the probability of all-cause mortality. The smooth curve fitting presented a Ushaped relationship between the LDL-C/HDL-C ratio and all-cause mortality. Adjusted for age, sex, BMI, SBP, DBP, TG, Hcy, FBG, SUA, eGFR, smoking, alcohol consumption, diabetes, stroke, CVD and anti-hypertensive drugs 
Table 3 The subgroup analysis for the LDL-C/HDL-C ratio on all-cause mortality

\begin{tabular}{|c|c|c|c|c|}
\hline \multirow[t]{2}{*}{ Subgroups } & \multicolumn{2}{|c|}{ Categories of the LDL-C/HDL-C ratio, HR (95\% CI) } & \multirow[b]{2}{*}{ High $(\geq 2.10)$} & \multirow[t]{2}{*}{$P$ for interaction } \\
\hline & Low $(<1.67)$ & Reference (1.67-2.10) & & \\
\hline Sex & & & & 0.249 \\
\hline male & $1.38(0.76,2.50)$ & 1 & $1.51(0.80,2.84)$ & \\
\hline female & $3.33(1.15,9.69)$ & 1 & $3.92(1.35,11.36)$ & \\
\hline $\mathrm{BMl}, \mathrm{kg} / \mathrm{m}^{2}$ & & & & 0.99 \\
\hline$<24$ & $1.81(1.01,3.26)$ & 1 & $2.13(1.14,3.98)$ & \\
\hline$\geq 24$ & $1.85(0.62,5.50)$ & 1 & $1.73(0.64,4.70)$ & \\
\hline Stroke & & & & 0.623 \\
\hline No & $1.71(1.00,2.91)$ & 1 & $1.81(1.05,3.14)$ & \\
\hline Yes & $3.63(0.40,32.93)$ & 1 & $6.89(0.79,60.40)$ & \\
\hline CVD & & & & 0.21 \\
\hline No & $1.58(0.93,2.69)$ & 1 & $2.01(1.17,3.45)$ & \\
\hline Yes & $4.23(0.43,41.60)$ & 1 & $1.38(0.10,18.38)$ & \\
\hline Diabetes & & & & 0.25 \\
\hline No & $1.63(0.95,2.79)$ & 1 & $1.68(0.96,2.94)$ & \\
\hline Yes & $6.38(0.80,50.66)$ & 1 & $8.18(1.04,64.02)$ & \\
\hline $\mathrm{eGFR}, \mathrm{ml} / \mathrm{min} / 1.73 \mathrm{~m}^{2}$ & & & & 0.097 \\
\hline$<60$ & $5.35(1.24,23.09)$ & 1 & $8.93(2.02,39.51)$ & \\
\hline$\geq 60$ & $1.45(0.82,2.55)$ & 1 & $1.44(0.80,2.57)$ & \\
\hline Smoking & & & & 0.452 \\
\hline No & $2.10(1.16,3.80)$ & 1 & $2.07(1.12,3.82)$ & \\
\hline Yes & $1.12(0.40,3.18)$ & 1 & $1.86(0.64,5.37)$ & \\
\hline Alcohol consumption & & & & 0.969 \\
\hline No & $1.75(1.01,3.03)$ & 1 & $1.99(1.13,3.48)$ & \\
\hline Yes & $1.87(0.40,8.68)$ & 1 & $1.91(0.38,9.68)$ & \\
\hline
\end{tabular}

Adjusted for age, sex, BMI, SBP, DBP, TG, Hcy, FBG, SUA, eGFR, smoking, alcohol consumption, diabetes, stroke, CVD and anti-hypertensive drugs, if not be stratified

suggested that the LDL-C/HDL-C ratio should be controlled above 2.96. Liu et al. [10] recruited 3250 stroke patients (mean age $63.72 \pm 11.33$ years, mean follow-up $=1.00$ years) and found a negative relationship between the LDL-C/HDL-C ratio and all-cause mortality. Mortality was lowest when the LDL-C/HDL-C ratio was between 2.23 and 2.88 . The findings of the above studies suggest that the relationship between the LDL-C/HDL-C ratio and all-cause mortality may be negative, and the proposed optimal range of the LDL-C/HDL-C ratio has been inconsistent. These conflicting results can be attributed to differences in the study populations, followup durations, and end-point events. Consequently, the exact relationship between the LDL-C/HDL-C ratio and all-cause mortality and the optimal LDL-C/HDL-C ratio are still unclear, which prompted us to conduct the current study.

The mechanism driving the association identified in this study remains unclear. Several possible mechanisms could explain the observed relationship. In this study, when the LDL-C/HDL-C ratio was low, there was a negative correlation between the LDL-C/HDL-C ratio and all-cause mortality. As shown in Table S2, we found that all deaths caused by respiratory diseases occurred in group of patients with LDL-C/HDL-C ratios < 1.67. A lower LDL-C/HDL-C ratio may be associated with inflammation, increasing the energy needed for respiration, which in turn would aggravate respiratory failure [16]. Notably, the lower LDL-C/HDL-C ratio was caused by higher HDL-C levels [17]. Consistent with our findings, Bowe et al. [18] included 1,764,986 US adults ( mean follow-up $=9.1$ years) and found a U-shaped relationship between HDL-C and all-cause mortality, suggesting that a higher HDL-C level was associated with increased mortality. C.M. Madsen et al. [19] included 116,508 patients (mean follow-up $=6.0$ years) and found that higher HDL-C levels were associated with increased all-cause mortality. It might be that a higher HDL-C level is associated with genetic variability, including mutations in ABCA1, LIPC, and SCARB1, which in turn 


\section{All-cause mortality}
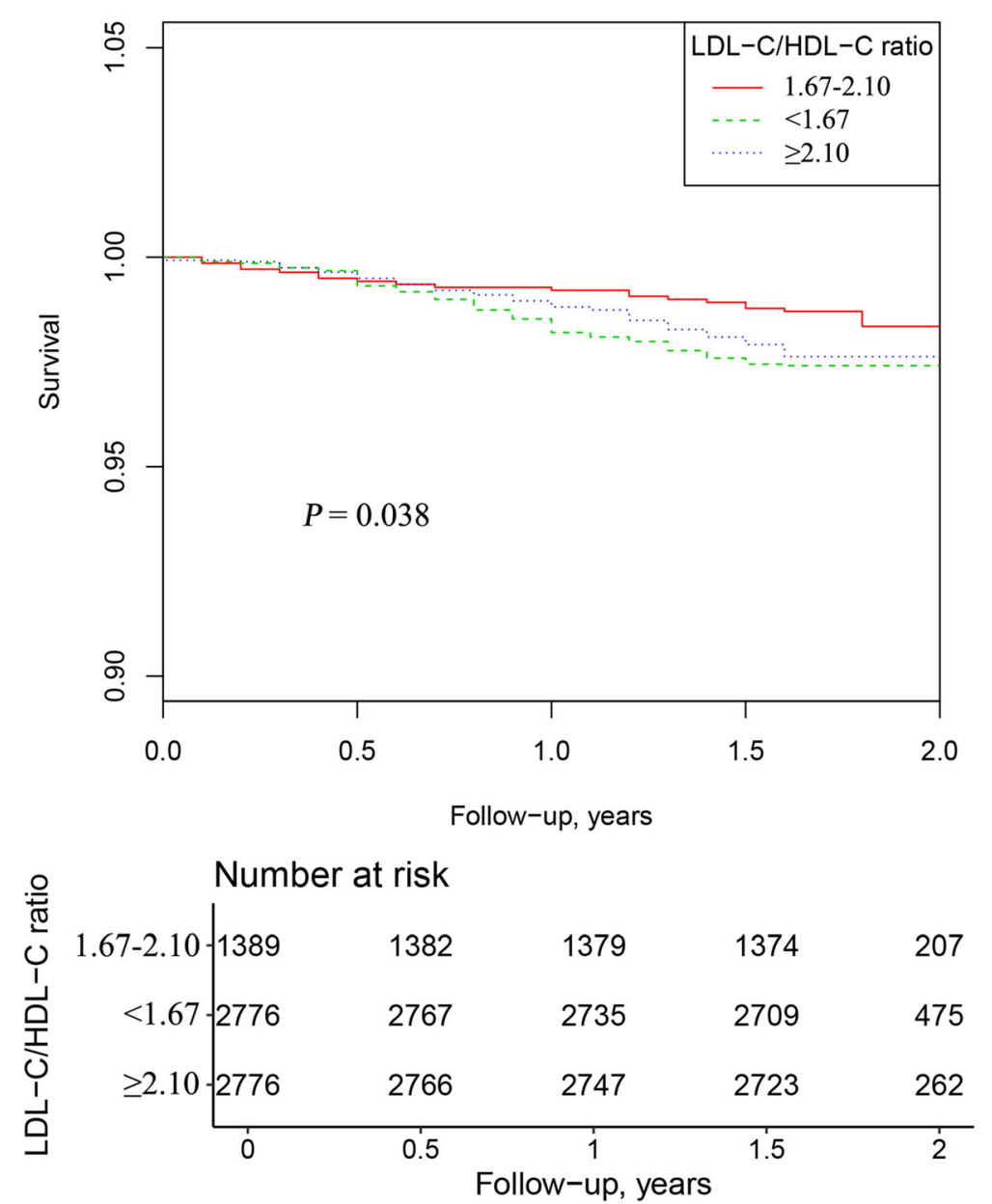

Fig. 2 Kaplan-Meier survival curve estimates for all-cause mortality in the elderly hypertensive population

promote the occurrence and progression of CVD [20,21]. However, HDL-C lost its protective effect at a higher level, paradoxically enhancing senescence and impairing endothelial progenitor cell vascularization and angiogenesis $[22,23]$. These mechanisms suggest that the conformational and functional properties of HDL particles may change when the level of HDL-C is high, which may lead to harmful effects. When the LDL-C/HDL-C ratio was high, it was positively associated with all-cause mortality. A higher LDL-C/HDL-C ratio may promote coronary inflammation [24], and may increase the vulnerability of coronary plaques to rupture $[24,25]$. In addition, a higher LDL-C/HDL-C ratio has been found to be associated with increased LDL-C levels [17]. Higher LDL-C levels have also been shown to be associated with increased mortality, mainly through mechanisms involving oxidative stress and the inflammatory response [26, 27]. Admittedly, all-cause mortality involves a variety of factors, so it is difficult to fully explain the mechanism underlying the relationship between the LDL-C/HDL-C ratio and all-cause mortality. Therefore, further basic experiments are needed to fully elucidate the specific biological mechanism underlying this connection.

Compared with the normal population, elderly hypertensive patients have higher mortality [28]. Therefore, it is more important to identify a valuable predictor of mortality in this population. The LDL-C/HDL-C ratio might be a valuable indicator that could be used for prognostic prediction because it simultaneously reflects the levels of both LDL-C and HDL-C. More importantly, a U-shaped relationship has a greater clinical significance than a linear relationship [29], because it suggests that there might be a target optimal range for the LDLC/HDL-C ratio.

\section{Study strengths and limitations}

This cohort study found a U-shaped relationship between the LDL-C/HDL-C ratio and all-cause mortality 
in elderly people with hypertension in a large sample population. Our findings provide a new therapeutic target for lipid-lowering therapy in elderly patients with hypertension. Nevertheless, some limitations should be noted. First, the present study did not analyze causespecific mortality due to the small sample sizes in the subgroups. However, this study is still ongoing, so the number of deaths will continue to increase. Second, our study population was elderly patients with hypertension, so the generality of our conclusions is limited. Third, we only analyzed the LDL-C/HDL-C ratio at baseline and did not observe dynamic changes over time. Finally, most of the evidence regarding the LDL-C/HDL-C ratio has come from studies performed in Asian populations, and large prospective cohort studies are needed to examine the predictive value of the LDL-C/HDL-C ratio for CVD or all-cause mortality in non-Asian populations.

\section{Conclusions}

The present study showed a U-shaped relationship between the LDL-C/HDL-C ratio and all-cause mortality in elderly hypertensive patients in China. Both lower and higher LDL-C/HDL-C ratios were associated with higher all-cause mortality. Compared with the lower and higher LDL-C/HDL-C ratio groups, patients with LDL-C/HDL$\mathrm{C}$ ratios of $1.67-2.10$ had a significantly higher survival probability. These results suggest that the LDL-C/HDL$\mathrm{C}$ ratio can be a valuable predictor of all-cause mortality in elderly hypertensive patients. For elderly patients with hypertension, the LDL-C/HDL-C ratio may be one of the targets of lipid-lowering therapy. Further large prospective studies are needed to confirm these findings and elucidate their clinical implications.

\section{Supplementary Information}

The online version contains supplementary material available at https://doi. org/10.1186/s12944-020-01413-5.

Additional file 1: Table S1. The study inclusion and exclusion criteria. Table S2. Mortality during follow-up was described according to classification of LDL-C/HDL-C ratio. Table S3. Association between the LDL-C/ $\mathrm{HDL}-\mathrm{C}$ ratio and all-cause mortality during the follow-up period. Table S4. The subgroup analysis for LDL-C/HDL-C ratio on all-cause mortality.

\section{Abbreviations \\ BMI: Body mass index; CVD: Cardiovascular disease; TC: Total cholesterol; TG: Triglyceride; LDL-C: Low density lipoprotein cholesterol; HDL-C: High density lipoprotein cholesterol; SUA: Serum uric acid; eGFR: Estimated glomerular filtration rate; Hcy: Homocysteine; FBG: Fasting blood glucose; HR: Hazard ratio; Cl: Confidence interval}

\section{Acknowledgements}

All authors acknowledge the contribution the all staff who participated in this study as well as the study participants who shared their time with us.

\section{Authors' contributions}

All authors were responsible for drafting the manuscript and revising it critically for constructive intellectual content. All authors approved the version to be published.

\section{Funding}

This study was supported by Science and Technology Innovation Platform Project of Jiangxi Province (Grant number: 20165BCD41005).

\section{Availability of data and materials}

The datasets generated and analyzed during the current study are not publicly available because this study is still on-going and the follow-up is not finished, but are available from the corresponding author on reasonable request.

\section{Ethics approval and consent to participate}

The study was conducted in accordance with the Declaration of Helsinki and was approved by the Ethics Committee of the Anhui Medical University Biomedical Institute (No. CH1059). Informed written consent was obtained from all patients before their enrollment in this study.

\section{Consent for publication}

All the authors have consented for the publication of this study.

\section{Competing interests}

The authors declare that they have no conflict of interest.

Received: 1 September 2020 Accepted: 2 November 2020

Published online: 12 November 2020

\section{References}

1. Zhou M, Wang H, Zeng X, Yin P, Zhu J, Chen W, Li X, Wang L, Wang L, Liu $Y$, et al. Mortality, morbidity, and risk factors in China and its provinces, 1990-2017: a systematic analysis for the global burden of disease study 2017. Lancet. 2019;10204:1145-58.

2. Wu X, Zhu B, Xu S, Bi Y, Liu Y, Shi J. A cross country comparison for the burden of cardiovascular disease attributable to tobacco exposure in China, , USA and world Japan. Bmc Public Health. 2020;1:888.

3. Enomoto M, Adachi H, Hirai Y, Fukami A, Satoh A, Otsuka M, Kumagae S, Nanjo Y, Yoshikawa K, Esaki E, et al. LDL-C/HDL-C ratio predicts carotid intima-media thickness progression better than $\mathrm{HDL}-\mathrm{C}$ or LDL-C alone. J Lipids. 2011;549137.

4. Ridker PM, Rifai N, Cook NR, Bradwin G, Buring JE. Non-HDL cholesterol, apolipoproteins A-I and B100, standard lipid measures, lipid ratios, and CRP as risk factors for cardiovascular disease in women. JAMA. 2005;3:326-33.

5. Yokokawa H, Yasumura S, Tanno K, Ohsawa M, Onoda T, Itai K, Sakata K, Kawamura K, Tanaka F, Yoshida Y, et al. Serum low-density lipoprotein to high-density lipoprotein ratio as a predictor of future acute myocardial infarction among men in a 2.7-year cohort study of a Japanese northern rural population. J Atheroscler Thromb. 2011;2:89-98.

6. Matsumoto I, Miyake Y, Mizukawa M, Takagi Y. Impact of low-density lipoprotein cholesterol/high-density lipoprotein cholesterol ratio on longterm outcome in patients undergoing percutaneous coronary intervention. Circ J. 2011:4:905-10.

7. Zhong Z, Hou J, Zhang Q, Zhong W, Li B, Li C, Liu Z, Yang M, Zhao P. Assessment of the LDL-C/HDL-C ratio as a predictor of one year clinical outcomes in patients with acute coronary syndromes after percutaneous coronary intervention and drug-eluting stent implantation. Lipids Health Dis. 2019;1:40

8. Kunutsor SK, Zaccardi F, Karppi J, Kurl S, Laukkanen JA. Is high serum LDL/ $\mathrm{HDL}$ cholesterol ratio an emerging risk factor for sudden cardiac death? Findings from the KIHD study. J Atheroscler Thromb. 2017;6:600-8.

9. You S, Zhong C, Xu J, Han Q, Zhang X, Liu H, Zhang Y, Shi J, Huang Z, Xiao $\mathrm{G}$, et al. LDL-C/HDL-C ratio and risk of all-cause mortality in patients with intracerebral hemorrhage. Neurol Res. 2016;10:903-8.

10. Liu L, Yin P, Lu C, Li J, Zang Z, Liu Y, Liu S, Wei Y. Association of LDL-C/HDL$C$ ratio with stroke outcomes within 1 year after onset: a hospital-based follow-up study. Front Neurol. 2020;408.

11. Williams B, Mancia G, Spiering W, Agabiti RE, Azizi M, Burnier M, Clement DL, Coca A, de Simone G, Dominiczak A, et al. 2018 ESC/ESH guidelines for the management of arterial hypertension. Eur Heart J. 2018;33:3021-104.

12. Yu Y, Hu L, Huang X, Zhou W, Bao H, Cheng X. BMI modifies the association between serum $\mathrm{HDL}$ cholesterol and stroke in a hypertensive population without atrial fibrillation. J Endocrinol Investig. 2020. 
13. 2018 Chinese Guidelines for Prevention and Treatment of Hypertension-A report of the Revision Committee of Chinese Guidelines for Prevention and Treatment of Hypertension. J Geriatr Cardiol. 2019;3:182-241.

14. Yu Y, Huang X, Li M, Ding C, Hu L, Zhong X, Zhou W, Wang T, Zhu L, Bao H, et al. Is there a nonlinear relationship between serum uric acid and lipids in a hypertensive population with eGFR $>/=30 \mathrm{ml} / \mathrm{min} / 1.73 \mathrm{~m}$ (2)? Findings from the China hypertension registry study. Int. J Endocrinol. 2020;9725979.

15. Gragnano F, Calabro P. Role of dual lipid-lowering therapy in coronary atherosclerosis regression: evidence from recent studies. Atherosclerosis. 2018:219-28.

16. Chio A, Calvo A, llardi A, Cavallo E, Moglia C, Mutani R, Palmo A, Galletti R, Marinou K, Papetti L, et al. Lower serum lipid levels are related to respiratory impairment in patients with ALS. Neurology. 2009;20:1681-5.

17. Awal HB, Nandula SR, Domingues CC, Dore FJ, Kundu N, Brichacek B, Fakhri M, Elzarki A, Ahmadi N, Safai S, et al. Linagliptin, when compared to placebo, improves CD34+ve endothelial progenitor cells in type 2 diabetes subjects with chronic kidney disease taking metformin and/or insulin: a randomized controlled trial. Cardiovasc Diabetol. 2020;1:72.

18. Bowe B, Xie Y, Xian H, Balasubramanian S, Zayed MA, Al-Aly Z. High density lipoprotein cholesterol and the risk of all-cause mortality among U.S. veterans. Clin J Am Soc Nephrol. 2016;10:1784-93.

19. Madsen CM, Varbo A, Nordestgaard BG. Extreme high high-density lipoprotein cholesterol is paradoxically associated with high mortality in men and women: two prospective cohort studies. Eur Heart J. 2017:32: 2478-86.

20. Agerholm-Larsen B, Nordestgaard BG, Steffensen R, Jensen G, TybjaergHansen A. Elevated HDL cholesterol is a risk factor for ischemic heart disease in white women when caused by a common mutation in the cholesteryl ester transfer protein gene. Circulation. 2000;16:1907-12.

21. Frikke-Schmidt R, Nordestgaard BG, Jensen GB, Steffensen $R$, TybjaergHansen A. Genetic variation in ABCA1 predicts ischemic heart disease in the general population. Arterioscler Thromb Vasc Biol. 2008;1:180-6.

22. Huang CY, Lin FY, Shih CM, Au HK, Chang YJ, Nakagami H, Morishita R, Chang NC, Shyu KG, Chen JW. Moderate to high concentrations of highdensity lipoprotein from healthy subjects paradoxically impair human endothelial progenitor cells and related angiogenesis by activating rhoassociated kinase pathways. Arterioscler Thromb Vasc Biol. 2012;10:2405-17.

23. Xiang AS, Kingwell BA. Rethinking good cholesterol: a clinicians' guide to understanding HDL. Lancet Diabetes Endocrinol. 2019;7:575-82.

24. Cushman M, Arnold AM, Psaty BM, Manolio TA, Kuller LH, Burke GL, Polak JF, Tracy RP. C-reactive protein and the 10-year incidence of coronary heart disease in older men and women: the cardiovascular health study. Circulation. 2005;1:25-31.

25. Kimura T, Itoh T, Fusazaki T, Matsui H, Sugawara S, Ogino Y, Endo H, Kobayashi K, Nakamura M. Low-density lipoprotein-cholesterol/high-density lipoprotein-cholesterol ratio predicts lipid-rich coronary plaque in patients with coronary artery disease--integrated-backscatter intravascular ultrasound study. Circ J. 2010;7:1392-8.

26. Yeboah J. Review: more-intensive vs less-intensive LDL-cholesterol lowering reduces mortality. Ann Intern Med. 2018;2:C6.

27. Benn M, Tybjaerg-Hansen A, Nordestgaard BG. Low LDL cholesterol by PCSK9 variation reduces cardiovascular mortality. J Am Coll Cardiol. 2019;24: 3102-14.

28. Chowdhury EK, Nelson MR, Wing L, Jennings G, Beilin LJ, Reid CM. Change in blood pressure variability among treated elderly hypertensive patients and its association with mortality. J Am Heart Assoc. 2019;21:e12630.

29. Hu L, Hu G, Xu BP, Zhu L, Zhou W, Wang T, Bao H, Cheng X. U-shaped Association of Serum Uric Acid with all-Cause and Cause-Specific Mortality in US adults: a cohort study. J Clin Endocrinol Metab. 2020;1.

\section{Publisher's Note}

Springer Nature remains neutral with regard to jurisdictional claims in published maps and institutional affiliations.

Ready to submit your research? Choose BMC and benefit from:

- fast, convenient online submission

- thorough peer review by experienced researchers in your field

- rapid publication on acceptance

- support for research data, including large and complex data types

- gold Open Access which fosters wider collaboration and increased citations

- maximum visibility for your research: over $100 \mathrm{M}$ website views per year

At BMC, research is always in progress.

Learn more biomedcentral.com/submissions 